\title{
Impresiones 3D de Cortes Transversales de un Cuerpo Humano: Un Recurso Didáctico para el Estudio de la Anatomía Seccional
}

\author{
3D Prints of Cross Sections of a Human Body: \\ A Teaching Resource for the Study of Sectional Anatomy
}

Denise Montt ${ }^{1}$; Iván Caro²; Andrés Neyem² \& Oscar Inzunza ${ }^{3}$

\begin{abstract}
MONTT, D.; CARO, I.; NEYEM, A. \& INZUNZA, O. Impresiones 3D de cortes transversales de un cuerpo humano: Un recurso didáctico para el estudio de la anatomía seccional. Int. J. Morphol., 38(3):578-584, 2020.

RESUMEN: Los cursos de anatomía constituyen un componente esencial del currículo de medicina, aportando las bases morfológicas para el examen clínico, la interpretación de imágenes médicas y la práctica segura de intervenciones quirúrgicas y procedimientos. Recientemente, la tecnología de impresión 3D ha permitido generar réplicas de disecciones de segmentos corporales a escala real que se utilizan como recursos docentes para el estudio de la anatomía humana, generando así modelos docentes de alta verosimilitud que sirven como alternativa al uso de preparaciones cadavéricas para la docencia anatómica. En este trabajo presentamos los resultados obtenidos al utilizar nuestro kit KAN3D que incluye réplicas físicas de secciones transversales del tronco y de las extremidades y una plataforma que aloja los modelos digitales debidamente rotulados, producto financiado con el proyecto FONDEF IT16I10073. La aplicación de estos productos en docencia señalan que las réplicas de secciones transversales de segmentos corporales presentan una alta verosimilitud en términos de forma, color, topografía y texturas, características que las validan como un excelente recurso docente para la docencia y el aprendizaje de la anatomía seccional humana. El kit KAN3D pone a disposición de los estudiantes de las carreras de la salud recursos de alta verosimilitud, disponibles a libre demanda, que les permita reproducir la experiencia de la actividad práctica de Morfología en el momento y lugar en que ellos se encuentren dispuestos, superando así las limitaciones de acceso a los pabellones de Anatomía y a material cadavérico de calidad.
\end{abstract}

PALABRAS CLAVE: Anatomía; Docencia; Impresión 3D; Plataforma digital.

\section{INTRODUCCIÓN}

Históricamente, los cursos de anatomía han constituido un componente esencial del currículo de medicina, aportando las bases morfológicas para el examen clínico, la interpretación de imágenes médicas y la práctica segura de intervenciones quirúrgicas y procedimientos (Inzunza, 2008; Davis et al., 2014; Smith et al., 2018). Este hecho aparece refrendado por un estudio de Rizzolo \& Stewart (2006), quienes señalan que aquellas escuelas de medicina que abandonaron o redujeron el estudio de la anatomía humana utilizando disección o prosección de material cadavérico han visto seriamente debilitadas las habilidades y destrezas clínicas de sus estudiantes. Una situación similar hemos detectado en nuestro medio, al analizar el rendimiento en pruebas de reconocimiento de estructuras anatómicas por parte de médicos de reciente graduación (Inzunza et al., 2007). Este hecho es interesante de considerar ya en nuestro país algunas escuelas de medicina han decidido abandonar el uso de material cadavérico para la docencia de los temas anatómicos, repitiendo así la fallida experiencia de ciertas universidades americanas denunciada hace más de 10 años por Rizzolo \& Stewart.

Recientemente, han aparecido trabajos que muestran la utilidad de la tecnología de impresión 3D para generar réplicas de disecciones de segmentos corporales que se utilizan como recursos docentes para el estudio de la anatomía humana (McMenamin et al., 2014; Lim et al, 2016; Smith et al. ). Asimismo, nosotros hemos utilizado esta tecnología de estéreo litografía para crear réplicas 3D de secciones transversales de tronco y de extremidades, material que hemos utilizado para el estudio de la anatomía humana seccional (Inzunza et al., 2015). Con esta tecnología de impresión $3 \mathrm{D}$ es posible reproducir, mediante escaneo de superficie o registro tomográfico $(\mathrm{CT})$, modelos de disecciones anató-

\footnotetext{
${ }^{1}$ Facultad de Arquitectura y Diseño, Universidad Católica de Chile, Santiago, Chile.

${ }^{2}$ Departamento de Ciencias de la Computación, Escuela de Ingeniería, Pontificia Universidad Católica de Chile, Santiago, Chile.

${ }^{3}$ Facultad de Medicina, Pontificia Universidad Católica de Chile, Santiago, Chile.
} 
micas, segmentos vasculares o de secciones transversales de un cuerpo humano a escala real, en materiales rígidos o semi rígidos, generando así modelos docentes de alta verosimilitud que sirven como alternativa al uso de preparaciones cadavéricas para la docencia anatómica (McMenamin et al.; Inzunza et al., 2015).

Por otro lado, está largamente probado que para el aprendizaje de temas morfológicos, el método consagrado por la historia es trabajo práctico con grupos pequeños, combinando el análisis de preparaciones cadavéricas y recursos imagenológicos (Chan \& Ganguly, 2008). Interesantemente, numerosas publicaciones han demostrado que las actividades prácticas en anatomía tienen un efecto nivelador sobre las diferencias de origen (culturales, económicas, sociales) con que ingresan los estudiantes; ya que en estas actividades se expone de manera igualitaria a los educandos a claves visuales de calidad que son relevantes para el proceso de aprendizaje de los temas morfológicos (Norvis, 1971; Bravo \& Inzunza, 1995; Inzunza \& Bravo, 2002; Biasutto et al., 2006; Inzunza et al., 2017), facilitando la conceptualización tridimensional de los distintos órganos y sistemas (Granger, 2004). Este dato contingente no es menor, ya que apunta a las políticas de equidad que promueven las instituciones de educación superior. A este respecto, resulta interesante considerar la evidencia internacional reciente que señala que el rendimiento en los cursos de anatomía constituye un excelente predictor en relación con el desempeño en el examen de licencia médica, el equivalente a nuestro examen médico nacional (Peterson \& Tucker, 2005; Tucker, 2008).

En este trabajo presentamos los resultados obtenidos al utilizar nuestro kit KAN3D (kit para el estudio de la anatomía humana seccional) en nuestros cursos de la carrera de medicina. Este recurso docente, generado con el financiamiento de nuestro proyecto FONDEF IT16i10073, incluye réplicas físicas de secciones transversales del tronco y de las extremidades y una plataforma que aloja los modelos digitales de los cortes cadavéricos debidamente rotulados, modelos virtuales que pueden ser rotados en 360 grados y ampliados por parte de los usuarios.

\section{MATERIAL Y MÉTODO}

En este trabajo, realizado en el Departamento de Anatomía de la Pontificia Universidad Católica de Chile, se analizan los resultados obtenidos por alumnos de la carrera de medicina en evaluaciones prácticas del curso de Morfología. En estas evaluaciones diseñadas de manera similar a una prueba tipo ECOE (coloquialmente llamadas Gymkanas) y bajo condiciones similares tiempo y de nivel de dificultad, los alumnos en cada estación deben identificar 4 elementos y escribir sus respuestas en una tablet en un tiempo total de 100 segundos. Se comparan los puntajes obtenidos por los alumnos de los cursos de Morfología, cohortes MED111, MED205 y MED212, en las evaluación prácticas del capítulo de tórax y del capítulo de aparato locomotor, miembro superior y miembro inferior.

Las evaluaciones estudiadas fueron:

Tórax P9: Identificación de elementos anatómicos en un corte real de tórax y en una réplica 3D del corte trazado a nivel de T7. Situación experimental: Los datos que presentamos corresponden a una evaluación práctica de reconocimiento de estructuras anatómicas en una preparación real (Fig. 1a, 63 alumnos) v/s la identificación de estructuras en la réplica 3D de la preparación de tórax, (Fig. 1b, 65 alumnos), realizada a un curso de la carrera de medicina; grupo que trabajó con el KAN3D en forma previa a la evaluación.
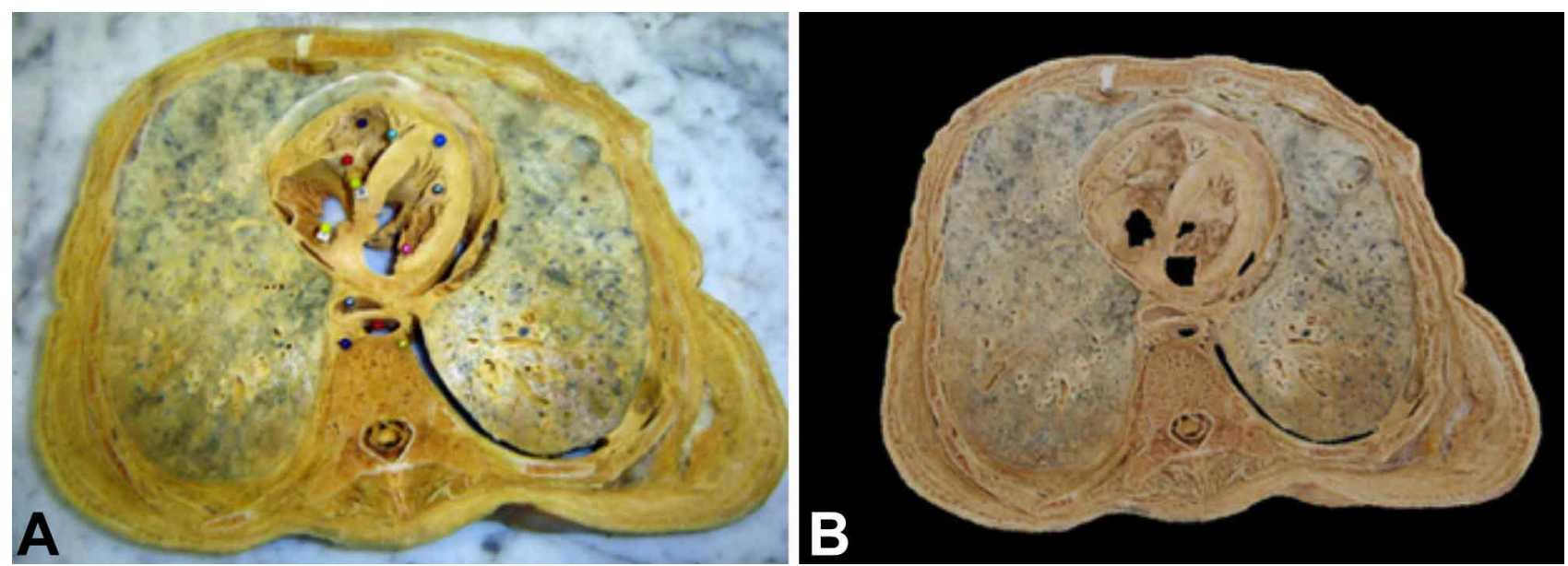

Fig. 1a. Sección transversal plastinada de tórax trazada a nivel de T7; 1b. Réplica 3D de la misma sección transversal. 
Miembro Superior: Identificación de estructuras anatómicas en secciones anatómicas reales plastinadas del tercio inferior de la diáfisis humeral. Situación experimental: Los datos que presentamos corresponden a evaluaciones prácticas de reconocimiento de estructuras anatómicas en un corte real de brazo (Fig. 2), realizadas a dos cursos de la carrera de medicina (MED205 y MED111; grupos de alumnos que no trabajaron con el KAN3D.

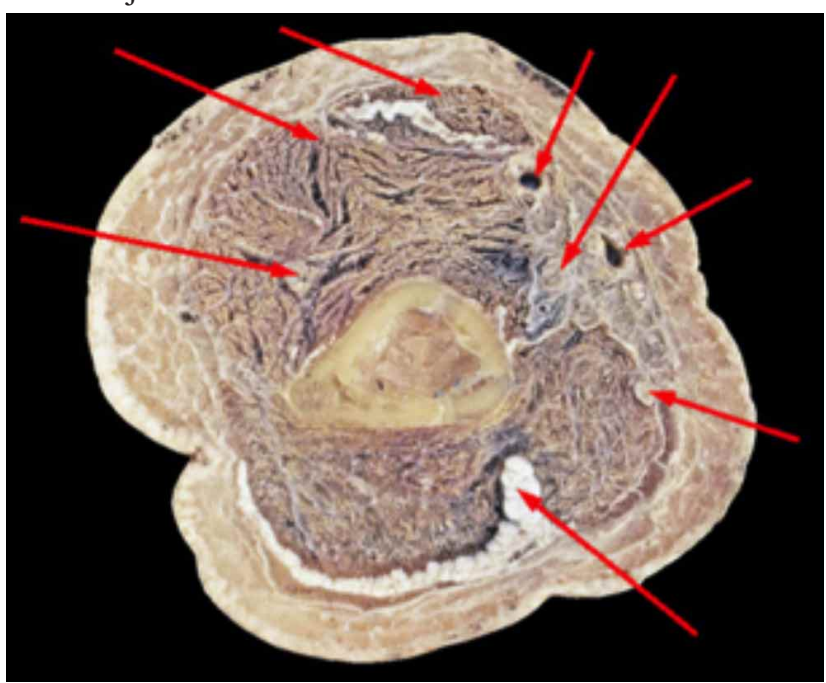

Fig. 2. Corte transversal del tercio inferior del brazo procesado mediante la técnica de plastinación.

Articulación de la Rodilla: Identificación de elementos anatómicos en un modelo 3D de la epífisis proximal de la tibia, réplica 3D de la preparación real que forma parte de nuestro kit KAN3D. Situación experimental: Los datos que presentamos corresponden a evaluaciones prácticas de reconocimiento de estructuras anatómicas en una réplica de la epífisis proximal de la tibia (Fig. 3), realizadas a dos cursos de la carrera de medicina; un curso trabajó con el material (MED111) y el otro no realizó esa experiencia (MED205).

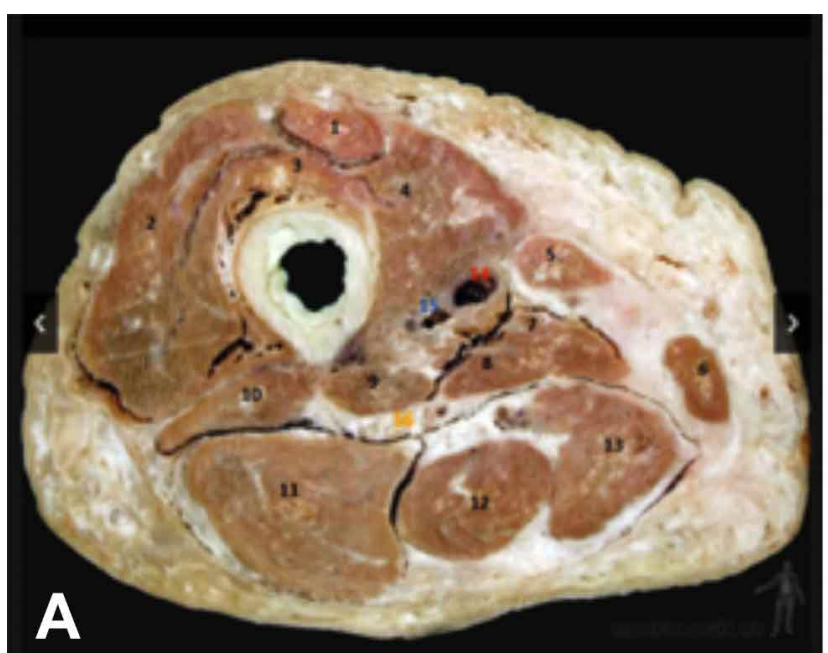

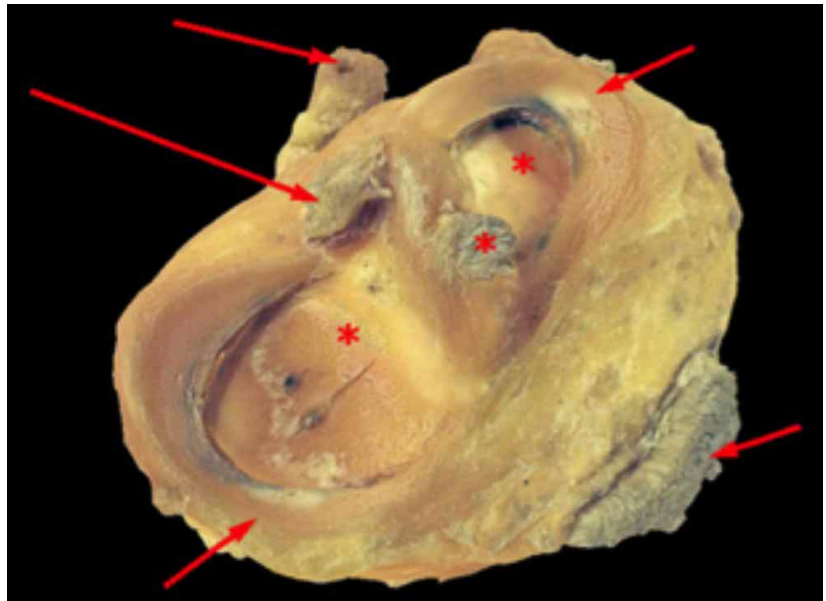

Fig. 3. Imagen de la vista superior de la réplica 3D de la epífisis proximal de la tibia.

Resonancia Magnética: Identificación de estructuras anatómicas en una imagen del tercio medio del muslo. Situación experimental: Los datos que presentamos corresponden a evaluaciones prácticas de reconocimiento de estructuras anatómicas en una resonancia nuclear magnética del tercio medio del muslo (Fig. 4b), realizadas a dos cursos de la carrera de medicina; un curso trabajó con el KAN3D (MED111, Fig. 4a) y otro no realizó esa experiencia (MED205).

\section{RESULTADOS}

A continuación presentamos los resultados obtenidos respecto de la utilidad docente de nuestro KAN3D (kit para el estudio de la anatomía humana seccional) en nuestros cursos de Morfología para la carrera de medicina. Este recurso do-

Fig. 4. A. Imagen virtual de una sección transversal de muslo, contenida en el KAN3D. 4b.- Imagen de una resonancia nuclear magnética del tercio medio del muslo utilizada en la evaluación. 
cente incluye réplicas físicas de secciones transversales del tronco y de los miembros y una plataforma que aloja los modelos digitales de los cortes cadavéricos debidamente rotulados.

\section{Identificación de elementos anatómicos en un corte real} de tórax y en una réplica 3D del corte trazado a nivel de T7. Los datos que presentamos corresponden a evaluaciones prácticas de reconocimiento de estructuras anatómicas en una preparación real (Fig. 1a) v/s la identificación de estructuras en la réplica de un corte de tórax (Fig. 1b), realizada a un curso de la carrera de medicina, cohorte MED212, grupo que trabajó con el KAN3D en forma previa (Tabla I). El grupo que identificó estructuras en la réplica corresponde a 65 alumnos y el grupo que fue evaluado en el corte real incluyen a 63 alumnos del curso.

El análisis estadísticos de los datos presentados en la Tabla I muestran que las diferencias entre los dos grupos MED212réplica y MED212real no son significativas $(\mathrm{P}=0,05)$.

Un dato importante a consignar en este experimento

Tabla I. Identificación de elementos anatómicos en un corte real de tórax y en una réplica 3D del corte trazado a nivel de T7.

\begin{tabular}{llll}
\hline MED2 12 réplica n:65 & & MED2 12 corte real n: 63 & \\
\hline & TóP9 & & TóP9 \\
Promedio & 2,523 & Promedio & 2,778 \\
Mediana & 3 & Mediana & 3 \\
Mínimo & 0 & Mínimo & 0 \\
Máximo & 4 & Máximo & 4 \\
& & & \\
Desviación estándar & 0,97 & Des viación estándar & 0,6826 \\
Error estándar & 0,1203 & Error estándar & 0,086 \\
\hline
\end{tabular}

Tabla II. Identificación de estructuras anatómicas en secciones anatómicas reales, sin haber trabajado con el KAN3D.

\begin{tabular}{llll}
\hline MED205 corte real n:124 & & MED1 11 corte real n: 124 \\
\hline & MSP8 & & MSP8 \\
Promedio & 3,64 & Promedio & 3,61 \\
Mediana & 4 & Mediana & 4 \\
Mínimo & 1 & Mínimo & 1 \\
Máximo & 4 & Máximo & 4 \\
& & & \\
Desviación estándar & 0,73 & Desviación estándar & 0,70 \\
Error estándar & 0,11 & Error estándar & 0,06 \\
\hline
\end{tabular}

Tabla III. Identificación de estructuras anatómicas en una réplica anatómica de la epífisis proximal de la tibia, modelo contenido en el KAN3D.

\begin{tabular}{llll}
\hline MED205 réplica n:124 & \multicolumn{3}{c}{ MED1 11 réplica n: 124 } \\
\hline Promedio & MI P7 & Promedio & MI0 \\
Mediana & 3,31 & Mediana & 3,69 \\
Mínimo & 4 & Mínimo & 4 \\
Máximo & 0 & Máximo & 0 \\
& 4 & & 4 \\
Des viación estándar & 0,90 & Desviación estándar & 0,77 \\
Error e stándar & 0,08 & Error estándar & 0,07 \\
\hline
\end{tabular}

El análisis estadísticos de los datos muestran que las diferencias entre los dos grupos MED205 y MED111 no son significativas $(\mathrm{P}=0,597)$. Un dato importante a consignar en este experimento es que, en términos generales, la disposición topográfica de los elementos anatómicos del tercio medio del brazo es relativamente sencilla, y para este grupo de alumnos las posibilidades de resolver el eje óseo central como el humero sin duda no ofrece grandes dificultades; el tema es deducir los compartimientos flexores y extensores con sus elementos y, en función de estos, identificar las estructuras finas interesadas y preguntadas en este corte. En definitiva, en un corte anatómico real, de complejidad media-baja, presentado sin tener referencias previas sobre este tipo de visualización del material, los alumnos como grupo no logran obtener una buena puntuación.

III. Identificación de estructuras anatómicas en una réplica anatómica de la epífisis proximal de la tibia, modelo contenido en el KAN3D. Los datos que presentamos corresponden a evaluaciones prácticas de reconocimiento de estructuras anatómicas en una réplica 3D de la epífisis proximal de la tibia (Fig. 3), realizadas a dos cursos de la carrera de medicina, un curso trabajó con el material MED 111, y otro no realizó esa experiencia MED 205 (Tabla III). 
El análisis estadísticos de los datos muestran que las diferencias entre las dos cohortes MED205 y MED111 son significativas $(\mathrm{P}=<0,001)$.

Un dato importante a consignar en este experimento es que este modelo 3D fue presentado por su vista superior (Fig. 3), y para reconocer las diferentes estructuras es relevante tener la posibilidad de manipularlo en 360 grados, particularmente en lo referido a la orientación mediolateral en la preparación. En definitiva, se trata de un modelo 3D complejo, que requiere de su manipulación para poder orientarse tridimensionalmente e identificar correctamente los distintos elementos anatómicos; los resultados de este experimento reflejan la utilidad de trabajar con el KAN3D, tanto con la réplica 3D como con el modelo virtual.

IV. Identificación de estructuras anatómicas en imágenes clínicas. Los datos que presentamos corresponden a evaluaciones prácticas de reconocimiento de estructuras anatómicas en una resonancia nuclear magnética del tercio medio del muslo (Fig. 4), realizadas a dos cursos de la carrera de medicina, un curso trabajó con el KAN3D y otro no realizó esa experiencia (Tabla IV).

El análisis estadísticos de los datos muestran que las diferencias entre los dos grupos MED205rmn y MED111rmn son significativas $(\mathrm{P}=<0,0001)$.

Un dato importante a consignar en este experimento es que la disposición topográfica de los elementos anatómicos del tercio medio del muslo es relativamente sencilla de reconocer en una imagen de RNM; sin embargo, para el grupo que nunca vio este tipo de presentación de la anatomía en secciones (MED205) se hace complejo de resolver, situación donde aparece aventajado el grupo que trabajó con el KAN3D (MED111). Estos validan a nuestro KAN3D como en un recurso docente que permite la transferencia de reconocimiento de estructuras en cortes al reconocimiento de elementos en imágenes radiológicas tomográficas.

Tabla IV. Identificación de estructuras anatómicas en imágenes clínicas.

\begin{tabular}{lclc}
\hline \multicolumn{2}{c}{ MED200 rmn } & \multicolumn{2}{c}{ MED111 rmn } \\
\hline Promedio & MI P3 & & MI P3 \\
Mediana & 2,483 & Promedio & 3,687 \\
Mínimo & 3 & Mediana & 4 \\
Máximo & 0 & Mínimo & 0 \\
Des viación estándar & 4 & Máximo & 4 \\
Error estándar & 1,152 & Desviación estándar & 0,7026 \\
& 0,1052 & Error estándar & 0,06138
\end{tabular}

\section{DISCUSIÓN Y CONCLUSIONES}

Las ciencias morfológicas, término que incluye a la anatomía, histología y embriología, corresponden a disciplinas donde las claves visuales en términos de forma, color, situación, topografía y textura son relevantes para la conceptualización tridimensional de los distintos tejidos, órganos y segmentos corporales (Norvis; Bravo \& Inzunza). Además, el aprendizaje de los temas morfológicos demanda de una manera novedosa el sensorio y la mente de nuestros noveles alumnos quienes, acostumbrados a responder preguntas de alternativas, deben enfrentarse ahora a reconocer y relacionar distintas estructuras en secciones histológicas, preparaciones cadavéricas o especímenes embriológicos tridimensionales (Inzunza et al., 2003).

En el área específica de la anatomía, hace casi ya dos siglos, Manuel Hurtado de Mendoza (1829) en su libro Tratado Elemental de Anatomía General ó Fisiológica, señalaba: "las preparaciones frescas y naturales son sin disputa el mejor medio que se haya empleado hasta ahora para apren- der anatomía", manifestando con esta frase la importancia del trabajo con cadáveres para la investigación de estos temas. Mas recientemente, Chan \& Ganguly señalan que está largamente probado que para el aprendizaje de temas morfológicos, el método consagrado por la historia es el trabajo práctico con grupos pequeños; gold estándar que combinado con el análisis imágenes clínicas permite mostrar importancia de la aplicación práctica del conocimiento anatómico en los currículos de las carreras del área médica.

En el último tiempo se han desarrollado métodos de impresión estereolitográfica de modelos de segmentos corporales utilizando imágenes de Tomografía Computarizada (Knox et al., 2005) o escáner de superficie (Hatamleh \& Watson, 2013; Inzunza et al., 2015); de modo que existe la tecnología para poder realizar réplicas $3 \mathrm{D}$ de secciones corporales o de disecciones humanas para aplicarlas con fines docentes. De hecho, recientemente esta tecnología se está incorporando al pabellón de anatomía (McMenamin et al.). 
En esta misma línea y con el financiamiento de nuestro proyecto FONDEF, hemos generado un nuevo recurso docente el kit KAN3D (kit para el estudio de la anatomía humana seccional), recurso docente que incluye las 31 réplicas físicas de las secciones transversales, material que logra copiar todos los detales de forma, relieve y color de la preparación original y, además, incluye todos los modelos digitales de los cortes cadavéricos debidamente rotulados; archivos que pueden ser recorridos para su visualización en pantalla en 360 grados. Es necesario recalcar que este recurso docente se ha generado a partir de secciones y preparaciones cadavéricas humanas plastinadas.

Analizando los resultados de la aplicación del novedoso recurso el kit KAN3D en nuestros cursos de morfología para la carrera de medicina podemos concluir:

1. Las secciones transversales de los distintos segmentos corporales presentan una alta verosimilitud, considerando las características físicas de forma, color, topografía y textura de las réplicas 3D (ver Figs. 1b y 3), características que las validan como un excelente recurso docente para la docencia y el aprendizaje de la anatomía seccional humana (Tabla I).

2. La utilización del recurso docente KAN3D, incluyendo las réplicas 3D y la plataforma docente, permite asentar en el alumno la conceptualización topográfica de distintos elementos interesados en los segmentos anatómicos. En efecto, el reconocer estructuras en un corte transversal del brazo (Fig. 2) representa un desafío importante a las cohortes MED111 y MED205, grupos que al no trabajar con este recurso docente que no exhiben diferencias en su rendimiento (Tabla II). Por el contrario, el utilizar el recurso KAN3D, tanto las réplicas físicas como los modelos virtuales facilita el reconocimiento de estructuras en preparaciones y modelos 3D de alta complejidad (Fig. 3 y tabla 3 ).

3. La utilización del recurso docente KAN3D, incluyendo las réplicas 3D y la plataforma docente, facilita la transferencia de reconocimiento de estructuras en cortes anatómicos al reconocimiento de elementos en imágenes radiológicas tomográficas (Tabla IV). En definitiva, el reconocimiento de estructuras en un corte anatómico real, en una réplica o en una imagen virtual (Figs. 1 a y b, 2, 3 y 4a) sin duda allana el camino para la visualización de elementos en imágenes clínicas, contribuyendo así a la conceptualización tridimensional de cuerpo humano.

4. Es interesante señalar que al abrir un espacio de diseño a través del modelado digital de material docente de alta verosimilitud basado preparaciones cadavéricas, se genera un producto docente complejo que podría complementarse con los atributos de los modelos de baja fidelidad; sirviendo como recursos de ayuda de memoria al simplificar características anatómicas complejas, reduciendo la sobrecarga cognitiva a la que pueden verse expuestos los estudiantes (Chan \& Cheng, 2011).

Finalmente, podemos agregar que en nuestro medio, donde anualmente ingresan al primer año de las carreras de la salud (profesionales y técnicas) más de 11.000 nuevos alumnos matriculados en más de 500 programas (CNA, 2015), muchos de los cuales no cuentan con el acceso a recursos docentes reales y de calidad para el aprendizaje de los temas morfológicos (anatomía, histología, embriología), resulta atractivo y deseable contar con nuevos recursos docentes, físicos y virtuales fidedignos, que traten de manera atractiva y unificada los temas morfológicos. El desafío es ofrecer a bajo costo el acceso a información relevante sobre estas temáticas. En definitiva, creemos que con nuestro producto KAN3D estamos colocando a disposición de los estudiantes de las carreras de la salud recursos de alta verosimilitud, disponibles a libre demanda, que les permita reproducir la experiencia de la actividad práctica de Morfología en el momento y lugar en que ellos se encuentren dispuestos, superando así las limitaciones de acceso a los pabellones de Anatomía y a material cadavérico de calidad. Con este desarrollo docente, aspiramos a que en este sistema universitario las diferencias en los atributos de los futuros profesionales esté fundada en las cualidades intrínsecas de las personas y no en las diferencias en el acceso a la información y a recursos docentes verosímiles (Inzunza et al., 2017).

Entonces, pasadas casi dos centurias de la edición del texto de Manuel Hurtado de Mendoza, donde señalaba: "Hay tres modos de estudiar la anatomía, ya en los libros, ya en el cadáver, ó ya á beneficio de las Fig.s imitadas en láminas, cera, etc.", ahora podemos agregar a esa sentencia otros recursos docentes como por ejemplo, las impresiones 3D y los modelos digitales de preparaciones anatómicas reales y la realidad virtual.

AGRADECIMIENTOS. Financiado por proyecto FONDEF IT16I10073.

MONTT, D.; CARO, I.; NEYEM, A. \& INZUNZA, O. 3D prints of cross sections of a human body: A teaching resource for the study of sectional anatomy. Int. J. Morphol., 38(3):578$584,2020$.

SUMMARY: Anatomy courses constitute an essential component of the medical curriculum, providing the morphological basis for the clinical examination, the interpretation of medical images and the safe practice of surgical interventions and procedures. Recently, 3D printing 
technology has allowed to generate replicas of dissections of body segments on a real scale that are used as teaching resources for the study of human anatomy, thus generating high-likelihood teaching models that serve as an alternative to the use of cadaveric preparations for Anatomical teaching. In this paper we present the results obtained by using our KAN3D kit that includes physical replicas of cross sections of the trunk and extremities and a platform that houses properly labeled digital models, a product financed with the FONDEF IT16I10073 project. The application of these products in teaching indicate that replicas of cross sections of body segments have a high likelihood in terms of shape, color, topography and textures, characteristics that validate them as an excellent teaching resource for teaching and learning the human sectional anatomy. The KAN3D kit makes available to students of health careers a high-likelihood resources, accesible on demand, that allows them to reproduce the experience of the practical activity of Morphology at the time and place where they are willing, exceeding thus the limitations of access to the Anatomy pavilions and quality cadaveric material.

Digital Platform.

KEY WORDS: Anatomy, Teaching, 3D Printing,

\section{REFERENCIAS BIBLIOGRÁFICAS}

Biasutto, S. N.; Caussa, L. I. \& Criado del Río, L. E. Teaching anatomy: cadavers vs. computers? Ann. Anat., 188(2):187-90, 2006

Bravo, H. \& Inzunza, H. O. Evaluación de algunos programas computacionales en la enseñanza de anatomía y neuroanatomía de la Facultad de Medicina de la Pontificia Universidad Católica de Chile. Rev. Chil. Anat., 13(1):79-86, 1995.

Chan, L. K. \& Cheng, M. M. W. An analysis of the educational value of low-fidelity anatomy models as external representations. Anat. Sci. Educ., 4(5):256-63, 2011.

Chan, L. K. \& Ganguly, P. K. Evaluation of small-group teaching in human gross anatomy in a Caribbean medical school. Anat. Sci. Educ., 1(1):1922, 2008.

Davis, C. R.; Bates, A. S.; Ellis, H. \& Roberts, A. M. Human anatomy: let the students tell us how to teach. Anat. Sci. Educ., 7(4):262-72, 2014.

Granger, N. A. Dissection laboratory is vital to medical gross anatomy education. Anat. Rec. B New Anat., 281(1):6-8, 2004.

Hatamleh, M. M. \& Watson, J. Construction of an implant-retained auricular prosthesis with the aid of contemporary digital technologies: a clinical report. J. Prosthodont., 22(2):132-6, 2013.

Hurtado de Mendoza, M. Tratado elemental completo de anatomía general ó [sic] fisiológica, de anatomía especial ó [sic] descriptiva, de anatomía de regiones ó [sic] quirúrgica y de anatomía patológica ó [sic] médica con arreglo al estado actual de esta ciencia y progresos que ha hecho en estos últimos años. Tomo 1. Madrid, Imprenta que fue de Garcia, 1829.

Inzunza, O. \& Bravo, H. Computer animation of photographs, an actual contribution to practical learning of human anatomy. Rev. Chil. Anat., 20(2):151-7, 2002.

Inzunza, O.; D'Acuña, E. \& Bravo, H. Practical evaluation in anatomy. Performance of first year medical students in relation to different ways of asking questions. Int. J. Morphol., 21(2):131-6, 2003.

Inzunza, H. O. Competencias generales en medicina, rol de la anatomía. Int. J. Morphol., 26(2):243-6, 2008.
Inzunza, O.; Vargas, A. \& Bravo, H. Anatomy and neuroanatomy the most impair in the curricular reform. Int. J. Morphol., 25(4):825-30, 2007.

Inzunza, O.; Caro, I.; Mondragón, G.; Baeza, F.; Burdiles, A. \& Salgado, G. 3D impressions, new technology that supports anatomical teaching. Int. J. Morphol., 33(3):1176-82, 2015.

Inzunza, O.; Schneeberger, D.; Salgado, G. \& Cantín, M. Practical activities in anatomy: leveling factor for the origin related contrasts among medical students. Int. J. Morphol., 35(2):494-9, 2017.

Knox, K.; Kerber, C. W.; Singel, S. A.; Bailey, M. J. \& Imbesi, S. G. Rapid prototyping to create vascular replicas from $\mathrm{CT}$ scan data: making tools to teach, rehearse, and choose treatment strategies. Catheter. Cardiovasc. Interv., 65(1):47-53, 2005.

Lim, K. H. A.; Loo, Z. Y.; Goldie, S. J.: Adams, J. W. \& McMenamin, P. G. Use of 3D printed models in medical education: a randomized control trial comparing 3D prints versus cadaveric materials for learning external cardiac anatomy. Anat. Sci. Educ., 9(3):213-21, 2016.

McMenamin, P. G.; Quayle, M. R.; McHenry, C. R. \& Adams, J. W. The production of anatomical teaching resources using three-dimensional (3D) printing technology. Anat. Sci. Educ., 7(6):479-86, 2014.

Norvis, G. Didáctica y Estructura de los Medios Audiovisuales. Buenos Aires, Kapeluz, 1971.

Peterson, C. A. \& Tucker, R. P. Medical gross anatomy as a predictor of performance on the USMLE step 1. Anat. Rec. B New Anat., 283(1):5$8,2005$.

Rizzolo, L. J. \& Stewart, W. B. Should we continue teaching anatomy by dissection when? Anat. Rec. B New Anat., 289(6):215-8, 2006.

Smith, C. F.; Tollemache, N.; Covill, D. \& Johnston, M. Take away body parts. An investigation into the use of 3D-printed anatomical models in undergraduate anatomy education. Anat. Sci. Educ., 11(1):44-53, 2018.

Tucker, R. P. Performance in a prematriculation gross anatomy course as a predictor of performance in medical school. Anat. Sci. Educ., 1(5):2247, 2008.

Dirección para Correspondencia:

Prof. Dr. Oscar Inzunza H.

Departamento de Anatomía

Facultad de Medicina

Pontificia Universidad Católica de Chile

Santiago

CHILE

Email: oinzunza@med.puc.cl

Recibido : 27-11-2019

Aceptado: 03-01-2020 